\title{
UM MODELO MATEMÁTICO APLICADO AO DIMENSIONAMENTO E SEQUENCIAMENTO DE LOTES EM MAQUINAS DISTINTAS EM PARALELO COM ESTOQUES INTERMEDIÁRIOS LIMITADOS
}

\author{
Gerson Ulbricht \\ IFSC - CEP 89254-430 - Jaraguá do Sul - SC. \\ e-mail: gerson.ulbricht@ifsc.edu.br \\ Neida M. Patias Volpi \\ UFPR - CEP 81531-990 - Curitiba - PR. \\ e-mail: neida@ufpr.br \\ Sander Joner \\ IFSC - CEP 89254-430 - Jaraguá do Sul - SC. \\ e-mail: sander.joner@ifsc.edu.br.
}

\begin{abstract}
RESUMO
Nesse trabalho é apresentado um modelo matemático de dimensionamento e sequenciamento de lotes com máquinas em paralelo composto por um estágio voltado à fabricação dos itens e outro ao teste de funcionamento, onde entre estes estágios, há a possibilidade de se manter um estoque intermediário de itens, o qual possui limitação de capacidade e custos de armazenamento. A problemática trata do sequenciamento e programação da produção dos itens nas máquinas paralelas bem como do envio desses itens para células de teste, onde estes são submetidos a testes de funcionamento por um tempo fixo em um ambiente com temperatura elevada. Neste artigo é proposto um modelo matemático voltado ao cumprimento da demanda estabelecida para o horizonte de planejamento, bem como pela otimização do uso das células de teste de carga, evitando o seu uso com espaços ociosos, de forma a reduzir custos na produção dos vários tipos de itens que compõe o mix de produção. Para validação do modelo, propõe-se uma aplicação com dados reais obtidos de uma empresa fabricante de itens eletrônicos o qual é resolvido com uso do software Cplex.
\end{abstract}

PALAVRAS CHAVE: Máquinas paralelas, estoques intermediários, programação da produção.

Área principal: Programação Matemática.

\begin{abstract}
This paper presents a mathematical model for batch sizing and scheduling with parallel machines consisting of a stage facing the manufacture of other items and the test run, where between these stages, there is the possibility of maintaining an intermediate stock items, which has limited capacity and storage costs . The problem is the sequencing and scheduling of items in parallel machines as well as sending these items to test cells, where items are subjected to functional testing for a fixed time in a high-temperature environment. This paper introduces one facing the implementation of an established demand for the planning horizon mathematical model, and by optimizing the use of cell load test, preventing its use with empty spaces in order to reduce costs in the production of various types of items that make up the production mix. To validate the model, we propose an application to real data obtained from a manufacturer of electronic items which is solved using CPLEX.
\end{abstract}

KEYWORDS: Parallel machines, intermediate stocks, production scheduling .

Main area: Mathematical Programming 


\section{INTRODUÇÃO}

Muitos problemas da engenharia possuem variáveis que podem ser ajustadas de tal maneira que possibilite um melhor aproveitamento de recursos e melhor lucratividade. Um desses problemas, ocorre no sistema industrial, onde coloca-se o sistema de planejamento, programação e controle da produção como uma área de decisão prioritária para as organizações. Realizar o planejamento do sequenciamento de tarefas implica melhorar o controle do fluxo da cadeia produtiva. Esse planejamento de tarefas deve ser feito de modo a permitir que a empresa consiga cumprir os prazos de entrega que foram acordados, sob o risco de perder clientes em caso de descumprimento. Além disso, deseja-se programar as atividades de tal maneira a utilizar os recursos disponíveis de uma forma mais eficiente possível. Os benefícios de um bom planejamento são o argumento da produtividade organizacional, redução dos custos de produção, agilidade no processo de tomada de decisão e aumento da eficiência organizacional. O problema de realizar sequenciamento de tarefas está presente em diversas áreas, como na indústria, na manufatura, no gerenciamento de processos de um computador, dentre outras, segundo Rego (2013).

O processo de dimensionar lotes de produção consiste em determinar quanto produzir de cada produto em cada período afim de atender uma demanda prevista sob as condições e capacidades operacionais existentes. Sequenciar lotes de produção significa determinar em que ordem produzir esses lotes de forma a melhor aproveitar os recursos produtivos e atender aos prazos estabelecidos.

De acordo com Pinedo (2008), os problemas de scheduling referem-se aos procedimentos de alocação, num determinado período de tempo, de recursos e equipamentos limitados para executar o processamento de tarefas. O scheduling possui três elementos fundamentais: a designação dos recursos que envolve a seleção de um conjunto de recursos para uma atividade conhecida; o sequenciamento de atividades, que define a ordem de execução das atividades designadas para os recursos; e a determinação dos tempos de início e término de cada atividade.

A complexidade dos problemas de dimensionamento de lotes dependem das características dadas no modelo. As características a seguir afetam a classificação, a modelagem e a complexidade das decisões no dimensionamento dos lotes, conforme citado por Karimi et al. (2003): horizonte de planejamento, número de níveis (estágios da produção), números de produtos, restrições de capacidade ou recursos, deterioração de itens,demanda,estrutura de preparação de máquina para produção (setup) e falta de estoque.

No Problema de Dimensionamento e Sequenciamento de Lotes Generalizado (General Lotsizing and Scheduling Problem - GLSP), cada período $t$ é subdivido em subperíodos de tamanho variável, fixando-se um número máximo de lotes a serem produzidos por período $\left(P_{t}\right)$. Cada lote está associado a um subperíodo (ou posição) no período $t$. Os subperíodos são indexados cronologicamente e de maneira contínua ao longo do horizonte de planejamento.

O GLSP, segundo Fleischmann \& Meyr (1997), é um modelo geral porque diversos modelos para dimensionamento e sequenciamento de lotes diferem dele apenas por restrições adicionais que modificam a estrutura e esforço computacional para obtenção das soluções.

O Problema de Dimensionamento de Lotes e Sequenciamento na Programação da Produção em Máquinas Paralelas (General Lotsizing and Scheduling Problem for Parallel Production Lines - GLSPPL) é um modelo análogo ao GLSP, onde os produtos são produzidos em um sistema de máquinas em paralelo e uma das decisões envolvidas é a alocação dos lotes de produção nas máquinas. Kang, Malik e Thomas (1999) trataram o GLSPPL considerando como função objetivo a minimização dos custos de setup e de estoque descontando-se o faturamento com as vendas.

Clark e Clark (2000) estudaram o GLSPPL cuja função objetivo minimiza custos de armazenagem e de backordering. Sua formulação matemática baseia-se numa premissa de que o número máximo de setups por período é pré-determinado. Foi utilizada uma heurística 
de horizonte rolante, que tratava exatamente um subproblema composto pelos períodos mais próximos, enquanto que o subproblema composto pelos períodos mais distantes era tratado relaxando-se a integralidade de suas variáveis. Uma heurística do tipo relax-and-fix é também utilizada nas variáveis que indicam as ocorrências de setup. Porém os resultados computacionais mostraram que somente problemas de pequeno porte puderam ser resolvidos em tempos factíveis.

Meyr (2002) adaptou a metodologia utilizada por ele mesmo em 2000, para abordar GLSPPL com máquinas distintas, visando minimizar os custos de produção, estoque e setup dependente sem permitir atrasos de entrega (backorders). O problema é resolvido através de metaheurísticas Threshold Accepting (TA) e Simulated Annealing (SA), que buscam padrões de setup (alocações dos lotes nas máquinas e seus respectivos sequenciamentos) de boa qualidade, e por um algoritmo de resolução de fluxo em rede que determina o tamanho dos lotes. Os testes foram realizados em instâncias reais de pequeno porte (19 produtos, 2 máquinas e 8 períodos) com máquinas idênticas, o que elimina a necessidade de custo diferenciado de produção na função objetivo.

Ferreira, Morabito e Rangel (2009) abordaram o problema de dimensionamento e sequenciamento de lotes com tempos e custos de setup dependentes em uma fábrica de refrigerantes, cujo sistema produtivo é composto de 2 estágios (preparação do refrigerante e engarrafamento) que devem ser sincronizados em linhas de produção em paralelo. Com o objetivo de minimizar os custos de estoque, de backordering e setup, foram desenvolvidos duas abordagens de resolução do problema que utilizavam 15 diferentes estratégias de relaxand-fix baseadas em conjugações de relaxação de variáveis de períodos, máquinas e estágios de produção.

Meyr e Mann (2013) apresentam uma nova solução heurística para o GLSPPL,que aborda o problema de, simultaneamente, decidir sobre os tamanhos e horários de lotes de produção em linhas de produção paralelas heterogêneas com relação à capacidade escassa, tempos de preparação dependentes da seqüência e demanda determinística e dinâmica de vários produtos. Seu objetivo é minimizar os custos de manutenção de estoque, de instalação e de produção dependente da sequência. A nova heurística iterativa decompõe o problema multi-linha em uma série de problemas de uma única linha, que são mais fáceis de resolver.

O presente trabalho trata do problema de dimensionamento e sequenciamento de lotes composto por máquinas paralelas, em um horizonte de planejamento de 1 dia de produção (8h), dividido em 4 períodos de $2 \mathrm{~h}$ cada, que é o tempo em que cada item necessita para ser testado em uma célula de teste com temperatura controlada. $O$ modelo trata do sequenciamento e programação da produção dos itens em 2 máquinas paralelas distintas onde os tempos de setup são dependentes da sequência dos itens a serem produzidos, considerando ainda, o envio dos itens para células de teste, onde estes são submetidos a testes de funcionamento. Trata-se neste trabalho do sequenciamento e programação da produção bem como da otimização do uso das células de teste de carga, evitando o seu uso com espaços ociosos, pois estas células são espécies de fornos e não podem ter seu ciclo interrompido para que seja adicionado um novo item e sendo assim, a falta de itens para completar sua capacidade máxima acarreta custos relacionados à subutilização deste estágio.

Esse trabalho está dividido como descrito a seguir: na seção 1 é apresentada a introdução e revisão de literatura. A seção 2 apresenta a descrição do problema em estudo. Na seção 3, é apresentada a metodologia do problema, sua formulação matemática e a implementação prática do modelo e na seção 4 são mostradas as principais conclusões do trabalho. 


\section{DESCRIÇÃO DO PROBLEMA}

Na problemática envolvida, tem-se um ambiente de produção composto por máquinas paralelas, em um horizonte de planejamento composto de períodos e subperíodos. Baseou-se a construção do modelo em uma fábrica de itens eletrônicos onde alguns desses itens podem ser produzidos ao mesmo tempo em diferentes máquinas, sendo que nem todas as máquinas, por limitações técnicas, são capazes de produzir todos os itens. O fato de se produzir um novo tipo de item em alguma das máquinas, gera um custo de setup, que é dependente da sequência, pois dependendo dos itens que se sucedem e/ou se antecedem, o setup pode ser complexo ou simples, pois acarreta na troca de componentes e ajustes nas máquinas.

Após produzidos os itens nas $M$ máquinas paralelas, esses são enviados a um setor de teste, denominado “Teste de Carga”, composto por células de teste, os quais são espécies de fornos que operam a uma temperatura elevada de modo a simular o funcionamento do produto em condições extremas, e que contam com conectores (slots) apropriados ao tipo de item que deve ser testado. Cada produto submetido a uma célula de teste deve ficar ali acondicionado por um tempo determinado de 2 horas, tempo este em que fica em operação simulando o real funcionamento. Existe na empresa um tipo diferente de célula para cada tipo de item, pois esta precisa estar adaptada ao item produzido devido aos conectores elétricos.

Um dos problemas encontrados, além do seqüenciamento e programação da produção nas $M$ máquinas paralelas, é o fato de tentar se trabalhar com as células de teste de forma que suas capacidades não sejam subutilizadas. Uma vez que uma célula de teste que tem capacidade para $N$ itens, começa a operar de forma a não estar completamente ocupada, o processo não pode ser interrompido pelo tempo de 2 horas. $\mathrm{O}$ fato de se esperar até que tenha o número necessário de itens para que o forno comece a operar gera estoque intermediário, causando transtorno devido às máquinas paradas e à imprevisão da chegada de novos itens. $\mathrm{O}$ fato da espera por novos itens até a completa lotação do forno, implica no processo de resfriamento/aquecimento ou da manutenção da célula de teste aquecida durante um tempo, o que gera custos. Devido ao tempo para aquecimento da célula, é mais viável manter a célula sempre aquecida durante o horizonte de planejamento.

É importante considerar que se não houver controle no processo de fabricação, podese ocasionar demora na produção de um determinado lote de itens ou ainda a produção seqüencial de um único tipo de item, o que causaria fila em determinada célula de testes enquanto que outras células permaneceriam ociosas. Um dos objetivos para qual o modelo matemático foi formulado, foi o de estabelecer um controle dos estoques intermediários evitando filas em determinadas células e falta de itens em outras, e ainda limitar a quantidade de itens em estoque intermediário. A figura 1 mostra uma idéia geral do ambiente de produção.

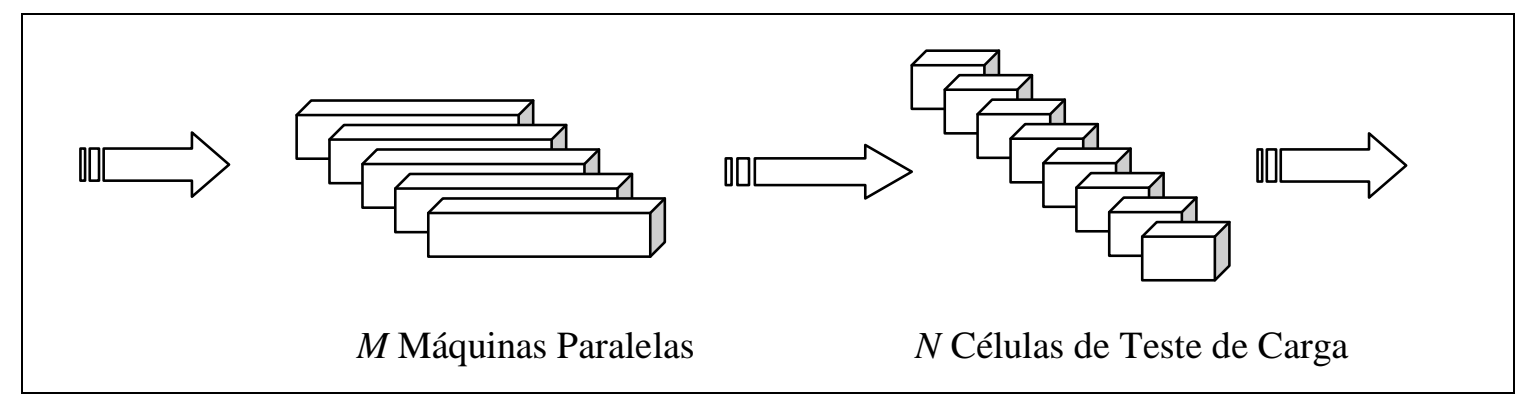

Figura 1: Esquema do ambiente de produção

Há de salientar o fato que nem sempre é possível produzir uma quantidade múltipla da capacidade da célula de teste de carga para um determinado item. Sendo assim, sempre que 
isso ocorrer, no modelo matemático foi aplicada uma penalidade por cada espaço ocioso cujo valor varia conforme o tipo de item, o que na prática está relacionado com os custos de operação de um espaço vazio em um determinado período. Como o modelo busca minimizar custos, faz com que os itens sejam produzidos sempre que possível de forma a utilizar o máximo possível da capacidade das células de teste e atender às demandas do período.

Neste problema, o horizonte de planejamento considera um dia de trabalho de 8 h, o qual foi dividido em 4 períodos de $2 \mathrm{~h}$ cada, pois este é o tempo necessário para testar o item nas células teste. Cada um dos períodos foi dividido em subperíodos, o que torna possível no modelo matemático, a produção de mais de um tipo de item por máquina em um mesmo período. A seção a seguir apresenta de forma detalhada e descrição do modelo matemático para este problema.

\section{METODOLOGIA}

Neste tópico é apresentado o modelo matemático, os dados referentes ao problema bem como os resultados encontrados e os cenários para diferentes números de itens.

\subsection{MODELO MATEMÁTICO}

O modelo proposto para o problema em estudo foi adaptado do modelo de programação inteira mista (MIP) de Meyr (2002), adicionando-se a permissão de atrasos de entrega e a restrição de armazenagem conforme proposto por Kawamura (2009).

Nesse modelo proposto pelos autores acima, o horizonte de planejamento é dividido simultaneamente em $T$ períodos e $W$ subperíodos, sendo que a cada período $t$ é constituído de subperíodos $s$. Em cada subperíodo permite-se a produção de apenas um tipo de produto, identificando um lote de produção. A divisão em subperíodos permite identificar a sequência de tarefas na máquina, assim como associar os respectivos custos e tempos de setup dependentes Kawamura (2009). De forma a otimizar a saída dos itens nas $M$ máquinas paralelas, neste trabalho estão sendo propostas novas restrições de modo a minimizar o estoque intermediário entre a saída das máquinas paralelas e a entrada nas células de teste, bem como maximizar a carga de cada célula de teste, sendo que para isso, foi inserido um custo (penalidade) por item, na operação de uma célula de teste com capacidade ociosa. A seguir é apresentado o modelo matemático para o problema em estudo.

\section{Parâmetros:}

$S P_{I}$ : conjunto de itens que podem ser produzidos na máquina $l$;

$D_{i}$ : demanda do item $i$ no horizonte de planejamento;

$C P_{l t}$ : capacidade de produção disponível na máquina $l$ no período $t$;

$c s_{i j l}$ : custo de setup para produzir o item $j$ imediatamente após o item $i$ na máquina $l$;

$c p_{i l}$ : custo de produção para produzir o item $i$ na máquina $l$;

$\square h_{i}$ : custo de manutenção de uma unidade do item $i$ não testado em estoque por período;

$\square p_{i l}$ : tempo consumido para produção de uma unidade do item $i$ na máquina $l$;

$\operatorname{lmin}_{i l}$ : lote mínimo do item $i$ produzido na máquina $l$.

$s t_{i j l}$ : tempo de setup para a produção do item $j$ imediatamente após o item $i$ na máquina $l$;

$I_{i 0}$ : quantidade de item $i$ não testado em estoque, no início do horizonte de planejamento;

$\square x_{i \text { ito }}: 1$, se a máquina está preparada para produzir o item $i$ na máquina $l$ no início do horizonte de planejamento; 0 , caso contrário;

$\square c f_{i}$ : custo da falta de cada unidade do item $i$ para completar a capacidade máxima da célula de teste;

$W_{t}$ : Conjunto de subperíodos contidos no período t; 
$C C T_{i}$ : capacidade da célula de teste para o item $i$;

CAT: capacidade de armazenamento de itens testados para todo o horizonte de planejamento;

$C A N T_{t}$ : capacidade de armazenamento de itens não testados para cada período $t$.

Variáveis de Decisão:

$q_{\text {ils }}$ : quantidade do item $i$ produzido na máquina $l$ no subperíodo $s$;

$x_{i l s}$ é igual a 1 , se a máquina 1 está preparada para produzir o item $i$ no subperíodo $s$; e 0 , caso contrário;

$y_{i j l s}$ é igual a 1 , se há setup do item $i$ para o item $j$ na máquina $l$ no subperíodo $s$; e 0 , caso contrário;

estoque no final do período $t$;

$I_{i t}$ : quantidade do item $i$ não testado em

$q f_{i t}$ : quantidade faltante para completar a capacidade máxima da célula de teste do item $i$ no período $t$;

$\square q c_{i t}$ : quantidade do item $i$ que será enviado à célula de teste dedicada a este item, no período $t$.

Modelo:

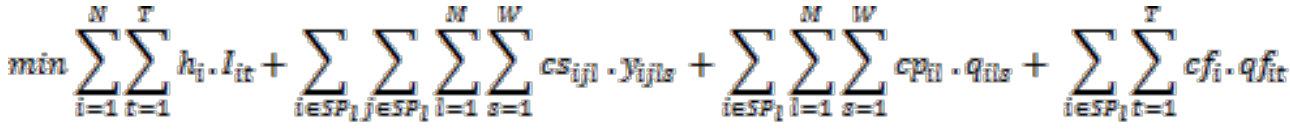

$$
\begin{aligned}
& I_{i, t-1}+\sum_{i=1}^{M} \sum_{s \in W_{t}} q_{i l s}-I_{\text {it }}=q c_{\text {it }} \\
& t=1_{, \ldots,} T \quad i \in S P_{1} \\
& \sum_{\mathrm{t}=1}^{T} \sum_{i=1}^{N} q c_{\mathrm{it}} \leq C A T \\
& \sum_{\mathrm{i}=1}^{N} I_{\text {it }} \leq C A N T_{\mathrm{t}} \\
& t=1_{s \ldots o} T
\end{aligned}
$$

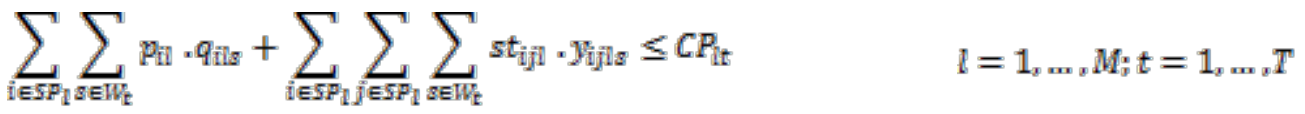

$$
\begin{aligned}
& p_{\text {ill }} \cdot q_{\text {ills }} \leq C P_{i \mathrm{t}} \times x_{\text {ills }} \\
& i \in S P_{1} ; l=1_{s, \ldots}, M ; t=1_{s, \ldots}, T ; s \in S_{\mathbb{I t}} \\
& q_{\mathrm{ils}} \geq \operatorname{lmin}_{\mathrm{in}} \cdot\left(x_{\mathrm{ills}}-x_{\mathrm{ills-1}}\right) \\
& i \in S P_{1} ; l=1_{v, \ldots}, M ; s \in W_{t} \\
& i=1, \ldots, N ; t=1, \ldots, T \\
& q c_{i t}+q f_{i t}=C C T_{i} \\
& \sum_{\mathrm{t}=1}^{T} q c_{\mathrm{it}}=D_{\mathrm{i}} \\
& i=1_{x \ldots} N \\
& \sum_{i \in S_{1}} x_{i l s}=1 \\
& l=1_{s \ldots}, M_{s} s \in W_{t} \\
& y_{i j l s} \geq x_{i l g-1}+x_{j l s}-1 \\
& i, j \in S P_{1} ; l=1_{s \ldots}, M ; s=1_{s \ldots}, W \\
& x_{\text {illo }}=0 \\
& x_{\text {ils }} \in\{0,1\}
\end{aligned}
$$




$$
\begin{gathered}
q c_{\text {it }}, I_{\text {it }}, q f_{\text {it: }} \text { inteiras } \\
Y_{\text {ijls }} \geq 0
\end{gathered}
$$

A função objetivo (1) a ser minimizada agrega os custos com armazenagem, setups, produção e custos de operação das células de teste com espaços ociosos. A restrição (2) realiza o balanço de estoques, relacionando quantidades produzidas, estoque intermediário de itens a serem testados com a capacidade da célula de teste para cada item por período em todo o horizonte de planejamento. A restrição (3) garante que a quantidade de itens que serão enviados para a célula de teste em todo o horizonte de planejamento não ultrapasse a capacidade de armazenamento de itens testados. A restrição (4) garante que a quantidade de itens não testados que compõem o estoque intermediário não exceda a capacidade de armazenamento de itens não testados. A restrição (5) garante a quantidade de horas consumidas na produção, adicionada da quantidade de horas necessárias para preparação da máquina, deve ser menor ou igual à capacidade disponível em cada máquina em cada período de tempo. A restrição (6) indica que somente haverá produção do item $i$ no subperíodo $s$ se a máquina estiver preparada para o item $\left(x_{i l s}=1\right)$. A restrição $(7)$ impõe uma condição de lote mínimo de produção para cada tipo de item em um subperíodo, que somente é ativada quando ocorre a preparação de máquina para um novo item. A restrição (7) permite impor uma quantidade mínima a ser produzida dentro de um período, de modo a compensar o acionamento de uma célula de teste. A restrição (8) impõe que a quantidade faltante para completar a capacidade máxima da célula teste do item $i$ no período $t$ é a diferença entre a capacidade da célula de teste para o item i e a quantidade do item i que será enviado à célula de teste dedicada a este item, no período $t$. A restrição (9) determina que a quantidade do item i que será enviado à célula de teste dedicada a este item deve ser igual à demanda do item i no final do horizonte de planejamento. A restrição (10) determina que a máquina esteja preparada para produzir somente um item em cada máquina em cada subperíodo $s$. A restrição (11) indica se houve mudança de produção do item $i$ para o item $j$ na máquina $l$ entre os subperíodos. A restrição (12) indica que as máquinas não estão preparadas para nenhum item no início do horizonte de planejamento $T$. A restrição (13) indica que a variável $x_{i l s}$ é binária e a restrição (14) indica que as variáveis $q c_{i t}, q_{i l s,} I_{i t}, y_{i j l s}, q f_{i l s}$ são reais não negativas.

\subsection{IMPLEMENTAÇÃO DO MODELO}

O modelo foi implementado em um caso prático numa indústria de cartões de circuito impresso, que trabalha simultaneamente com 8 tipos de itens. Esses itens são produzidos em 2 máquinas paralelas, chamadas de SMD (Surface Mount Device), onde alguns dos itens podem ser produzidos ao mesmo tempo em diferentes máquinas, sendo que nem todas as máquinas, por limitações técnicas, são capazes de produzir todos os itens. Após a produção nestas máquinas, cada cartão é submetido a um teste de carga, onde este é conectado a equipamentos elétricos, ficando operando por $2 \mathrm{~h}$ em um ambiente com temperatura elevada e controlada. Devido às especificidades de cada item, há uma célula de teste, que é um compartimento onde é possível acondicionar um certo número de itens, sendo que essa quantidade é diferente para cada item. Isso se deve às particularidades de cada item a ser testado, como por exemplo, a tensão e corrente elétrica a que é submetido, aos slots para conexão, ao tamanho físico e à temperatura a que este deve ser testado.

Sendo assim, cada item possui uma célula de teste específica na qual será testado. Neste caso, principal problema em questão é saber em que momento produzir cada tipo de item que consta na demanda do dia, de modo a reduzir custos de produção no bloco de máquinas paralelas, bem como evitar acumular itens em algumas células enquanto outras operam com espaços ociosos ou ainda permanecem aquecidas à espera de itens. 
Nas tabelas a seguir são apresentados os parâmetros utilizados para a resolução do problema, os quais foram obtidos de uma empresa que fabrica itens eletrônicos, como teste piloto para a aplicação do modelo.

\begin{tabular}{c|ccccc}
\hline Item & $\begin{array}{c}\text { Demanda } \\
\left(D_{i}\right)\end{array}$ & $\begin{array}{c}\text { Capacidade } \\
\text { da célula de } \\
\text { teste }\left(C C T_{i}\right)\end{array}$ & $\begin{array}{c}\text { Custo da } \\
\text { falta na } \\
\text { célula de } \\
\text { teste }\left(c f_{i}\right)\end{array}$ & $\begin{array}{c}\text { Estoque } \\
\text { inicial de } \\
\text { itens não } \\
\text { testados }\left(I_{i 0}\right)\end{array}$ & $\begin{array}{c}\text { Custo de estoque } \\
\left(h_{i}\right)\end{array}$ \\
\hline Item 1 & 28 & 10 & 3 & 0 & 4 \\
Item 2 & 22 & 6 & 7 & 0 & 3 \\
Item 3 & 30 & 8 & 8 & 0 & 3 \\
Item 4 & 15 & 4 & 7 & 0 & 3 \\
Item 5 & 16 & 5 & 6 & 0 & 4 \\
Item 6 & 20 & 6 & 6 & 0 & 4 \\
Item 7 & 19 & 6 & 7 & 0 & 4 \\
Item 8 & 18 & 5 & 6 & 0 & 3 \\
\hline
\end{tabular}

Tabela 1: Parâmetros para cada item.

\begin{tabular}{|c|c|c|c|c|}
\hline & & $\begin{array}{l}\text { Custo de produção } \\
\left(\subset p_{\text {ifi }}\right)\end{array}$ & $\begin{array}{l}\text { Tempo de produção } \\
\left(p_{\text {iD }}\right)\end{array}$ & $\begin{array}{l}\text { Lote mínimo por } \\
\text { item } i\left(\operatorname{lmin}_{i \eta}\right)\end{array}$ \\
\hline \multirow{2}{*}{ Item 1} & Máq. 1 & 8 & 6 & 2 \\
\hline & Máq. 2 & 9 & 8 & 2 \\
\hline \multirow{2}{*}{ Item 2} & Máq. 1 & 7 & 4 & 2 \\
\hline & Máq. 2 & 9 & 4 & 2 \\
\hline \multirow{2}{*}{ Item 3} & Máq. 1 & 7 & 4 & 3 \\
\hline & Máq. 2 & 7 & 4 & 3 \\
\hline \multirow{2}{*}{ Item 4} & Máq. 1 & 5 & 2 & 2 \\
\hline & Máq. 2 & 4 & 2 & 2 \\
\hline \multirow{2}{*}{ Item 5} & Máq. 1 & 6 & 4 & 2 \\
\hline & Máq. 2 & 5 & 6 & 2 \\
\hline \multirow{2}{*}{ Item 6} & Máq. 1 & 9 & 6 & 1 \\
\hline & Máq. 2 & 8 & 6 & 1 \\
\hline \multirow{2}{*}{ Item 7} & Máq. 1 & 6 & 6 & 2 \\
\hline & Máq. 2 & 5 & 10 & 2 \\
\hline \multirow{2}{*}{ Item 8} & Máq. 1 & 5 & 4 & 1 \\
\hline & Máq. 2 & 6 & 8 & 1 \\
\hline
\end{tabular}

Tabela 2: Parâmetros para cada item em diferentes máquinas.

No problema estudado a capacidade de armazenamento de itens testados para todo o horizonte de planejamento $C A T$ foi fixada em 300 itens. A capacidade de armazenamento de itens não testados para cada período $t\left(C A N T_{t}\right)$ foi, para os quatro períodos de respectivamente 80 itens.

No modelo em estudo, todos itens podem ser produzidos em qualquer uma das máquinas, o que foi definido pelas variáveis $\left(S P_{l}\right)$. Cada período $t$ foi dividido em 8 subperíodos para fins de modelagem do problema, sendo importante salientar que após a resolução e análise dos resultados, a duração de um subperíodo na prática, é dada pela quantidade produzida de cada item no subperíodo s, multiplicada pelo tempo de produção do respectivo item, o que torna os subperíodos de tamanho variável. Todas as máquinas estavam preparadas para a produção, no início do horizonte de planejamento. A capacidade de produção $C P_{l t}$ (em minutos) disponível em cada máquina $l$ por período $t$ é apresentada na tabela 3.

\begin{tabular}{c|cccc}
\hline & Período 1 & Período 2 & Período 3 & Período 4 \\
\hline Máq. 1 & 120 & 120 & 120 & 60 \\
Máq. 2 & 120 & 120 & 120 & 100 \\
\hline \multicolumn{4}{c}{ Tabela 3: Capacidade de produção disponível em cada máquina l }
\end{tabular}


Na tabela 4, é mostrado o custo de setup ( $\left.c s_{i j l}\right)$ e tempo de setup ( $\left.s t_{i j l}\right)$ para produzir o item $j$ imediatamente após o item $i$ na máquina $l$.

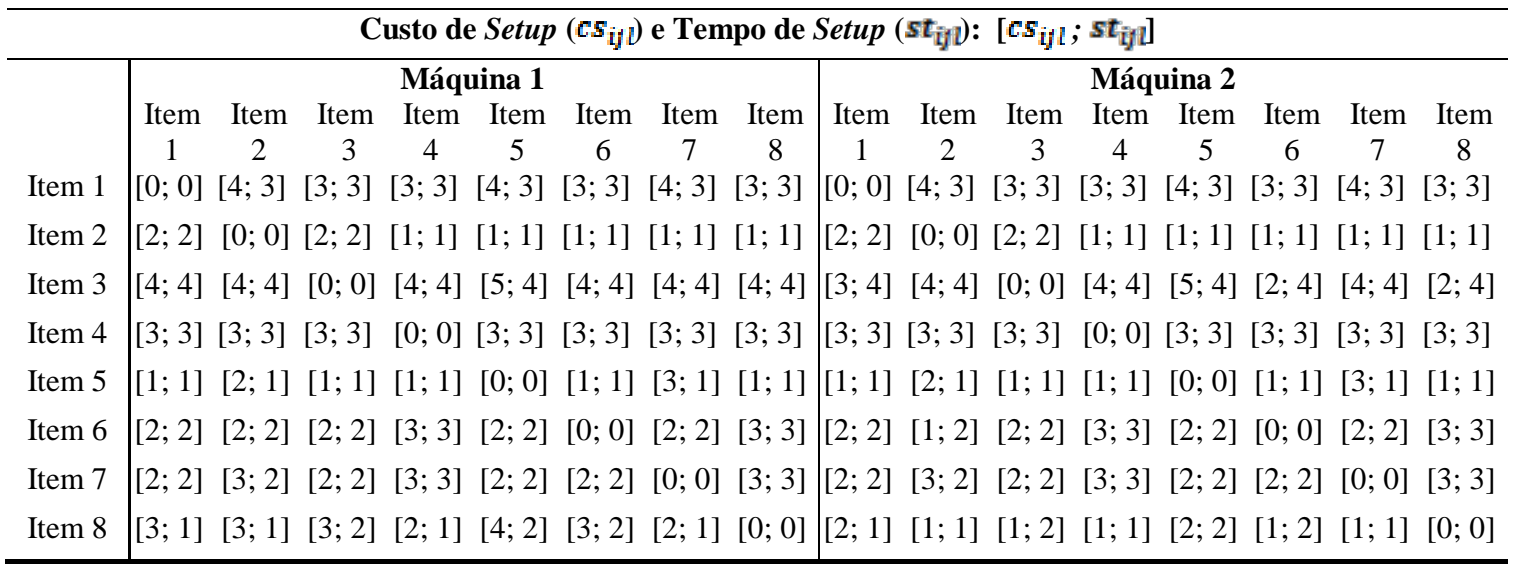

Tabela 4: Custo e tempo de setup dependente da sequência em cada máquina.

O modelo foi resolvido utilizando IBM ILOG CPLEX Optimization Studio 12.6 com interface OPL. Para execução do modelo foi utilizado um computador com processador Intel Core i5 (2M Cache, 1,7GHz) e 8Gb de memória RAM.

Devido ao elevado tempo computacional verificado, para obtenção de uma solução inteira bem como da solução ótima, a qual não foi encontrada devido às dimensões do problema, optou-se por utilizar uma heurística de resolução baseado na relaxação das variáveis inteiras.

Assim, na obtenção de uma solução viável para o problema foi utilizada a heurística Relax-and-fix (Mercé e Fontan (2003); Kawamura (2009)), a qual se baseia na partição das variáveis inteiras do problema criando $P$ conjuntos distintos, $Q_{i}, \mathrm{i}=1, \ldots, P$, sendo que o número $P$ de conjuntos determina o número de iterações da heurística (Moraes, 2012). Ainda conforme Moraes (2012), “em uma iteração $n$, apenas as variáveis do conjunto $Q_{n}$ são definidas como inteiras e as demais variáveis inteiras são relaxadas ou fixadas”.

A tabela 5, apresenta os resultados obtidos na execução do modelo matemático com a heurística Relax-and-fix, considerando os parâmetros apresentados nas tabelas 1 a 4.

\begin{tabular}{|c|c|c|c|c|c|c|c|c|c|}
\hline Período & Descrição & Item 1 & Item 2 & Item 3 & Item 4 & Item 5 & Item 6 & Item 7 & Item 8 \\
\hline \multirow{4}{*}{$\begin{array}{c}\text { Período } \\
1\end{array}$} & Quantidade produzida Máq. 1 & 10 & 0 & 0 & 0 & 0 & 0 & 6 & 5 \\
\hline & Quantidade produzida Máq. 2 & 0 & 4 & 8 & 4 & 3 & 6 & 0 & 0 \\
\hline & Quant. faltante para completar célula & 0 & 2 & 0 & 0 & 2 & 0 & 0 & 0 \\
\hline & Quant. em estoque intermediário & 0 & 0 & 0 & 0 & 0 & 0 & 0 & 0 \\
\hline \multirow{4}{*}{$\begin{array}{c}\text { Período } \\
2\end{array}$} & Quantidade produzida Máq. 2 & 0 & 0 & 8 & 4 & 3 & 5 & 2 & 0 \\
\hline & Quant. enviada à célula de teste & 8 & 6 & 8 & 4 & 3 & 6 & 5 & 4 \\
\hline & Quant. faltante para completar célula & 2 & 0 & 0 & 0 & 2 & 0 & 1 & 1 \\
\hline & Quant. em estoque intermediário & 0 & 0 & 0 & 0 & 0 & 0 & 0 & 0 \\
\hline \multirow{3}{*}{$\begin{array}{c}\text { Período } \\
3\end{array}$} & Quant. enviada à célula de teste & 8 & 6 & 8 & 4 & 5 & 4 & 6 & 5 \\
\hline & Quant. faltante para completar célula & 2 & 0 & 0 & 0 & 0 & 2 & 0 & 0 \\
\hline & Quant. em estoque intermediário & 0 & 0 & 0 & 0 & 0 & 0 & 0 & 0 \\
\hline Período & Quantidade produzida Máq. 1 & 2 & 4 & 0 & 0 & 0 & 0 & 2 & 4 \\
\hline
\end{tabular}


Tabela 5: Resultados obtidos na execução do modelo matemático.

Na aplicação da heurística procedeu-se de forma que em um primeiro momento foi mantida a condição de integridade para as variáveis $q_{i l s}$, e $I_{i t}$ pertencentes ao primeiro período das máquinas 1 e 2 e relaxadas as integridades destas mesmas variáveis nos demais períodos. Depois de encontrados os valores correspondentes a estas variáveis no primeiro período, estes valores foram fixados como parâmetros e então as variáveis $q_{i l s}$, e $I_{i t}$ das máquinas 1 e 2 do segundo período foram declaradas como inteiras e as dos períodos subseqüentes foram relaxadas e assim sucessivamente até completar o último período.

Os valores das variáveis $q_{\text {ils }}$ (quantidade do item $i$ produzido na máquina $l$ no subperíodo $s$ ), obtidos após a resolução do problema, possibilitam verificar as quantidades produzidas em cada subperíodo bem como a ordem em que essas tarefas serão realizadas. Esses resultados foram ilustrados e são apresentados no quadro 1, o qual mostra o sequenciamento da produção nas duas máquinas considerando as quantidades de cada item conforme apresentado na tabela 5, e o sequenciamento das tarefas segundo os resultados obtidos das variáveis $q_{i l s}$. Os dados se referem ao produto das quantidades fabricadas em cada subperíodo pelo tempo de produção $\left(q_{i l s} \cdot p_{i i}\right)$ de cada item, bem como o tempo de setup entre itens. O tempo em que a máquina fica ociosa até o início do próximo período é representada por “Oc” nesta ilustração.

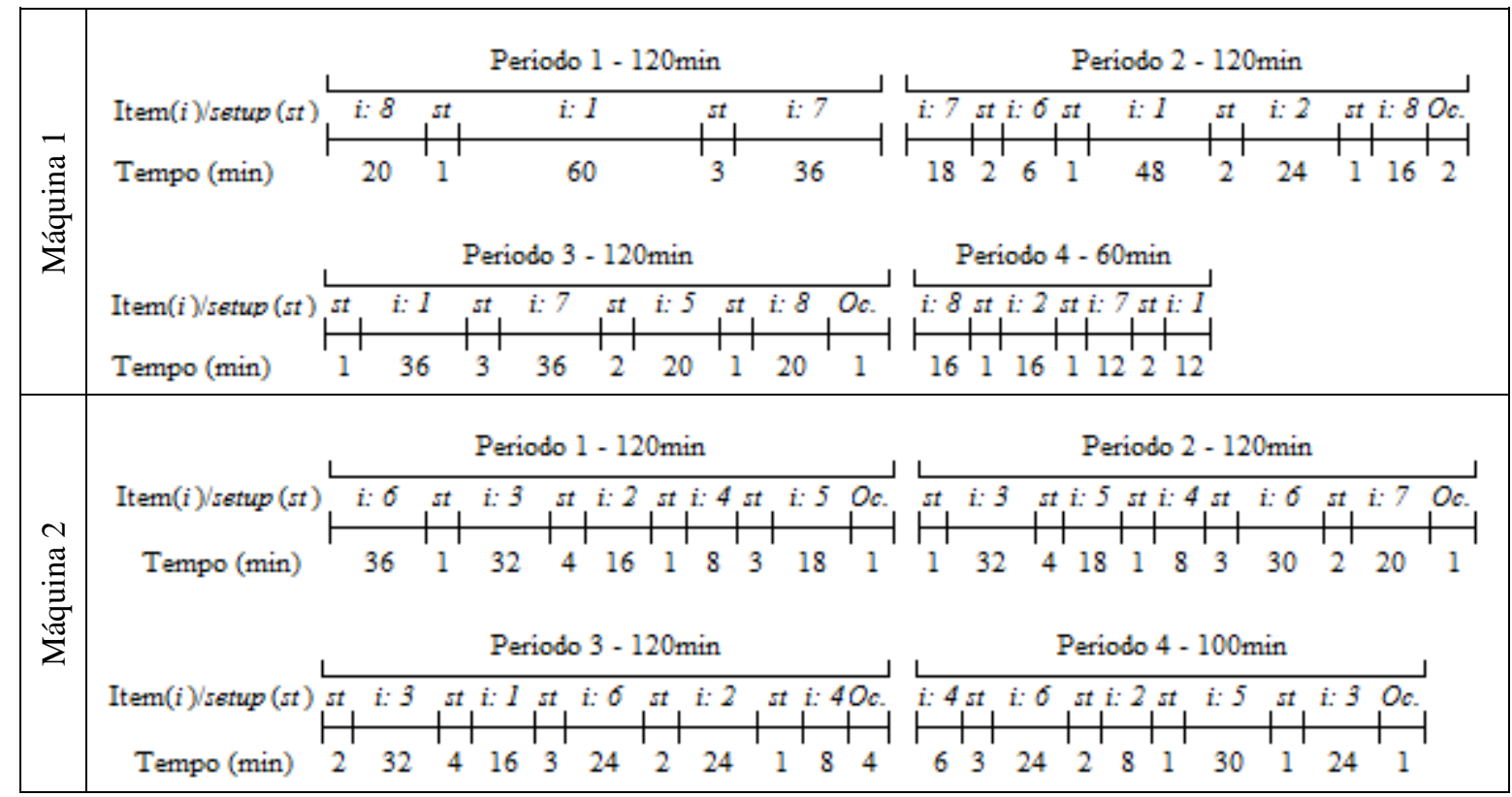

Quadro 1: Sequenciamento de tarefas em cada máquina

Como resultado por meio do Relax-and-fix para esta aplicação no ambiente industrial foi obtida função objetivo no valor de 1.360,00, onde, deste total tem-se: custo de setup de 70,00 , custo de produção 1.122,00, custo com teste de carga 168,00, não havendo custo de armazenagem já que nenhum item foi estocado.

Os resultados obtidos apresentam uma boa estratégia para ser aplicada na empresa no que se refere ao sequenciamento e programação da produção. Observa-se que na empresa estudada, tal procedimento é realizado considerando apenas o planejamento manual por parte da gerência de produção, e a implantação de um modelo de otimização tende a trazer significativos avanços no planejamento das tarefas de modo a cumprir as demandas estabelecidas minimizando custos operacionais. 


\section{CONCLUSÕES}

O principal objetivo neste trabalho foi o fato de estabelecer um modelo matemático que fosse capaz de atender ao atual cenário da linha de produção em um caso específico de uma indústria de itens eletrônicos. Testes realizados com os dados reais da empresa, mostraram que o modelo matemático está formulado adequadamente, atendendo às necessidades da programação de produção e trazendo resultados satisfatórios.

O modelo matemático apresentado neste artigo apresentou-se bem adaptado para a resolução do problema de otimização dos recursos e tempo.

Resultados promissores que heurísticas de resolução podem trazer, conduziram os autores a estudarem outros métodos que possam trazer boas soluções em um tempo computacional razoável. Alguns testes com heurísticas baseadas principalmente em estratégias de relaxamento de variáveis inteiras, destacando-se a Relax-and-fix, estão sendo realizados atualmente pelos autores para este modelo e poderão ser objeto de publicações futuras.

\section{REFERÊNCIAS BIBLIOGRÁFICAS}

[1] Bitran, G.R, Yanasse, H.H. Computational complexity of the capacitated lot size problem. Management Science,28-10,pp. 1174-1186,1982.

[2] Clark,A., Clark,S. Rolling-horizon lot-sizing when set-up times are sequence dependent.International Journal of Production Research, 38,pp. 2287-2307,2000.

[3] Ferreira, D., Clark, A. R., Almada-Lobo, B., Morabito, R.. Single-stage formulations for synchronised two-stage lotsizing and scheduling in soft drink production. International Journal of Production Economics 136 (2), 255-265, 2012

[4] Ferreira D, Morabito R, Rangel S. Solution approaches for the soft drink integrated production lot sizing and scheduling problem. Eur J Oper Res 196(2):697-706, 2009.

[5] Fleischmann, B.; Meyr, H. The general lotsizing and scheduling problem. OR Spektrum, v. 19 n. 1, p. 11-21, 1997.

[6] Kang, S.,Malik, K.,Thomas,L.J. Lotsizing and scheduling on parallel machines with sequence-dependent setup costs. Management Science,45-2,pp.273-289,1999.

[7] Karimi, B., Fatemi Ghomi S.M.T., Wilson, J.M. The capacitated lotsizing problem: a review of models and algorithms. Omega 31, 365-378, 2003.

[8] Kawamura, S. M. Aplicação da Heurística Relax-and-Fix no Problema de Dimensionamento e Sequenciamento de Lotes de Produção em Máquinas Distintas em Paralelo. Anais do XLII SBPO. Bento Gonçalves, 2009.

[9] Mercé, C. e Fontam, G. Mip-based heuristics for capacitated lotsizing problems. International Journal of Production Economics, 85, 97-111, 2003.

[10] Moraes, L. C. C.; Santos M. O. Heurísticas Relax-and-fix para o Problema de Dimensionamento de Lotes com Janelas de Tempo de Execução. Anais do Congresso Latino Iberoamericano de Investigación Operativa. Rio de Janeiro, 2012.

[11] Meyr, H. Simultaneous lotsizing and scheduling on parallel machines. European Journal of Operational Research, 139, pp.277-292, 2002.

[12] Meyr H., Mann, M. A decomposition approach for the General Lotsizing and Scheduling Problem for Parallel production Lines. European Journal of Operational Research, 229,pp. 718-731,2013.

[13] Pinedo, M. L. Scheduling: theory, algorithms, and systems. Springer Verlag. $3^{\mathrm{a}}$. Ed. , 2008.

[14] Rego,M.F. Algoritmos Multiobjetivos para o Problema de Sequenciamento de Tarefas em Uma Máquina com Tempo de Preparação Dependente da Sequência e da 
Família.Dissertação de Mestrado, Programa de Pós-Graduação em Ciência da Computação,Universidade Federal de Ouro Preto,Ouro Preto, 2013.

[15] Schrage, L. Order allocation for stock cutting in the paper industry. Operations Research, 50, pp. 324-332, 2002. 